\title{
Pediatric follicular lymphoma of the parotid gland
}

\author{
Jangyoun $\mathrm{Choi}^{1}$, \\ Hyuk Joon Choi ${ }^{1}$, \\ Kwangil Yim², \\ Ho Kwon ${ }^{1}$, \\ Jun Hee Byeon ${ }^{1}$, \\ Sung-No Jung ${ }^{1}$ \\ Departments of ${ }^{1}$ Plastic and \\ Reconstructive Surgery and ${ }^{2}$ Pathology, \\ Uijeongbu St. Mary's Hospital, College \\ of Medicine, The Catholic University of \\ Korea, Uijeongbu, Korea
}

\begin{abstract}
Primary malignant lymphoma rarely presents as a mass in the salivary gland. It accounts for about $1 \%$ of salivary gland tumors. The lymphomas of the parotid gland are mainly non-Hodgkin's lymphoma of B-cell follicular type. It usually occurs in male adults and is very rare in children. In contrast to the intractable disease course of adult parotid follicular lymphoma, when occurred in children or adolescent, its prognosis is very good when it is first treated with surgical excision. Thus, a solitary follicular lymphoma in an extranodal site that has occurred in children is termed separately as pediatric follicular lymphoma (PFL). We share our treatment experience of a 16-year-old PFL patient through surgical removal combined with superficial parotidectomy. In line with the few previous case reports of PFL, we suggest that active surgical removal should be undertaken for solitary, extranodal follicular lymphoma of the pediatric and adolescent population.
\end{abstract}

Keywords: Follicular lymphoma / Malignancy / Parotid neoplasms

\section{INTRODUCTION}

Salivary gland neoplasms in children are rare. Among those neoplastic disorders, malignancies of the salivary glands in children and adolescents are particularly rare, with an estimated annual incidence of 0.08 per 100,000 children [1]. Mucoepidermoid carcinoma is the most common malignant parotid neoplasm in children, followed by acinic cell carcinoma and lymphoma. Primary malignant lymphoma of parotid gland in children is diagnosed in about $1.7 \%$ of patients with parotid neoplasms [2].

We are reporting a case of extranodal, follicular subtype of parotid gland lymphoma in an adolescent, which is separately termed as pediatric follicular lymphoma (PFL). A 16-year-old adolescent presented with asymptomatic and unilateral parotid swelling of a 4-month duration, slowly progressing in size. The patient underwent superficial parotidectomy and was diag-

\footnotetext{
Correspondence: Sung-No Jung

Department of Plastic and Reconstructive Surgery, Uijeongbu St. Mary's Hospital, College of Medicine, The Catholic University of Korea, 271 Cheonbo-ro, Uijeongbu 11765 , Korea

E-mail: jsn7190@catholic.ac.kr

Received August 14, 2018 / Revised October 18, 2018 / Accepted November 21, 2018
}

nosed as PFL on final pathology. The patient was tolerable without complication or recurrence.

\section{CASE REPORT}

A 16-year-old male patient presented to our institution for evaluation of a slowly growing, nontender, 4-cm-sized firm mass over the left parotid gland area (Fig. 1). He did not complain of any mass-related symptoms. No systemic symptoms, such as fever, night sweat, and weight loss were present. A computed tomography (CT) scan showed a $3.5 \times 2.0$-cm-sized, homogenous soft tissue density lesion in the superficial lobe of left parotid gland (Fig. 2). Surgical removal was planned under the impression of common parotid tumors such as pleomorphic adenoma or Warthin's tumor.

Mass removal with superficial parotidectomy was performed with routine precautions to preserve the facial nerve and the parotid duct. Operative wound healed uneventfully. Routine hematoxylin and eosin stain showed extensive proliferation of follicular cells (Fig. 3). To further differentiate the diagnosis from various lymphoproliferative or inflammatory disorders of the parotid gland, immunohistochemical (IHC) and molecular 


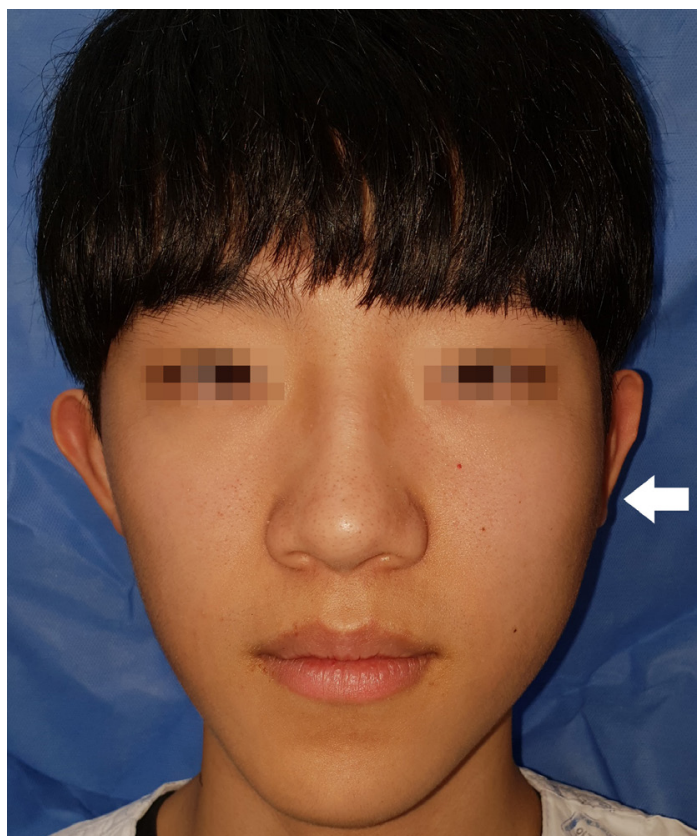

Fig. 1. Clinical photo at initial presentation. A firm mass measured about $4 \mathrm{~cm}$ was found on the left parotid gland area (arrow).

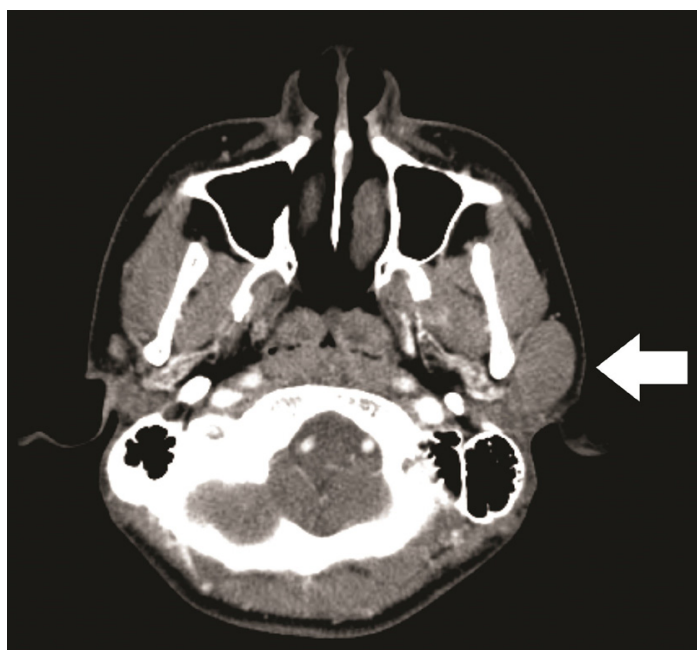

Fig. 2. Preoperative computed tomography (CT) scan. CT showed a $3.5 \times 2.0$-cm-sized soft tissue density lesion in the superficial lobe of left parotid gland (arrow).

pathology were done.

Typical follicular lymphoma (FL), Mantle cell lymphoma and marginal zone lymphoma were excluded by IHC stain for CD10, CD20, Bcl2, CD3, CD5, and Ki-67 (Fig. 4). Reactive follicular hyperplasia was ruled out by molecular pathology looking into B-cell monoclonality. Considering the patient's age, the site of onset, and the patterns of IHC stain, PFL was confirmed as the final diagnosis.

Further cancer workup was undertaken for staging of the disease. Peripheral blood cell counts and bilateral bone marrow

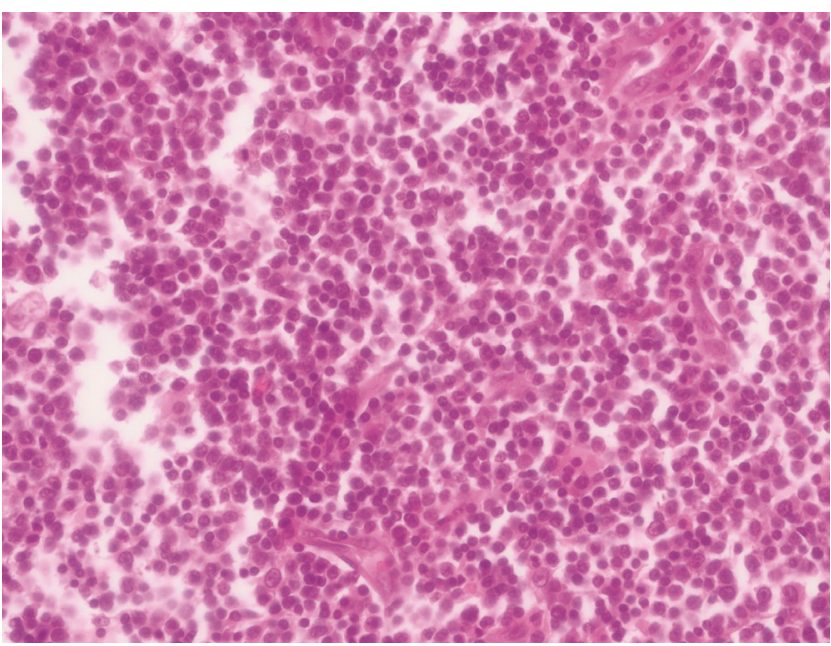

Fig. 3. Massive follicular hyperplasia was observed in histologic analysis $(\mathrm{H} \& \mathrm{E}, \times 400)$.
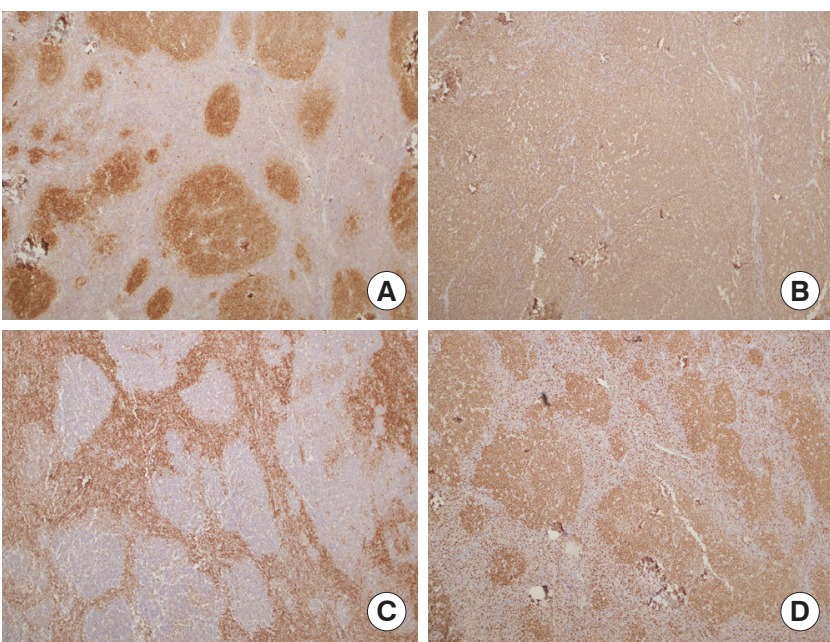

Fig. 4. Immunohistochemical stain. (A) CD10 and (B) CD20 (B-cell markers) were positive. This finding is consistent with follicular $\mathrm{B}$-cell presence. (C) $\mathrm{Bcl} 2$ was negative in follicular area, which is a characteristic difference between adult type and pediatric follicular lymphoma. (D) A reactive pattern was observed in Ki-67 stain, suggesting malignant proliferation (all panels, $\times 100$ ).

biopsies were negative for malignancy. On positron emission tomography-CT (PET-CT), there was no evidence of additional lymph node involvement. For prevention of recurrence, the patient received 12 cycles of local radiation therapy to the site of the primary tumor after 2 months from surgery (planning target volume 1, 24 Gy; planning target volume 2, 21.6 Gy). Six months after surgery, follow-up CT scan showed no recurrence of the tumor (Fig. 5).

\section{DISCUSSION}

In this case, we described a primary parotid lymphoma in a 


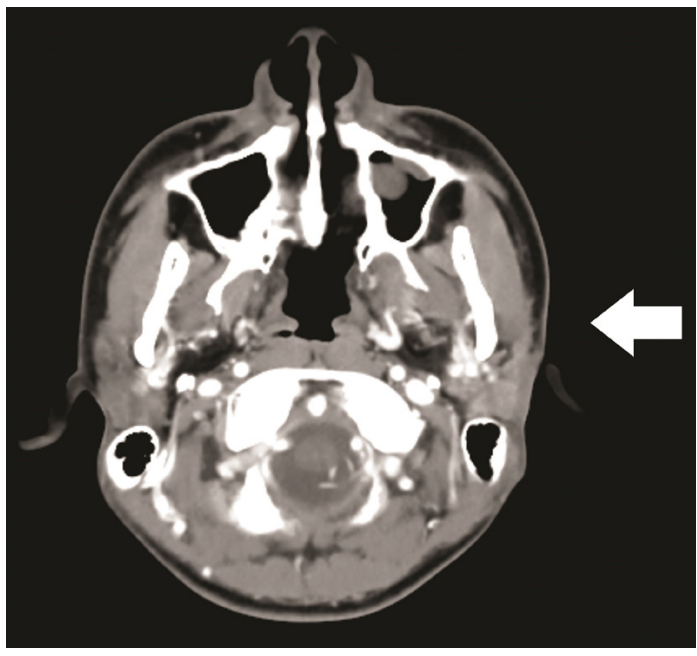

Fig. 5. Postoperative follow-up computed tomography after 6 months. No tumor recurrence was found (arrow).

16-year-old male patient. Regardless of age, malignant parotid neoplasm is very uncommon. Lymphoma in salivary glands shows the lowest incidence, representing $1.7 \%-5 \%$ of all reported salivary neoplasms [2]. Considering the fact that extranodal lymphoma is usually diagnosed in the sixth decade of life, the occurrence of parotid lymphoma in the pediatric population is the rarest of all. Only sporadic case reports describe primary parotid lymphoma occurring in middle-aged adults [3-5]. In our case, histologic subtype revealed FL, which is a Bcell origin non-Hodgkin lymphoma. FL arising in extranodal sites, or presenting as a localized lymphadenopathy in a child or adolescent is termed PFL.

PFL is considered as a variant of conventional FL [6]. The histologic and molecular difference between two categories has not been clearly elucidated yet. However, the distinction between conventional FL and PFL is important because of its course and prognosis. Conventional FL, which occurs in adults, is known to have an indolent but incurable clinical course. This presents at a later stage and usually requires intensive systemic chemotherapy after resection [7,8]. However, pediatric FL shows excellent treatment outcome, sometimes with surgical excision alone [9]. Therefore, surgery should always be considered as first-line treatment for solitary PFL.

At preoperative phase, PFL of the parotid gland is mostly indistinguishable from other common parotid neoplasms. Without the hallmark B symptoms (fever, night sweat, weight loss) of lymphoma, the patient merely complains of a nontender and soft mass on the cheek. Preoperative CT shows homogenous soft tissue density, which is undiscernible from other common parotid neoplasms. Semi-invasive diagnostic measures such as fine needle aspiration provides insufficient information because of the minuscule sample available from the procedure. Staging workups include routine laboratory workup, bone marrow biopsy, and PET-CT. Usually, the final diagnosis is made after complete surgical removal of the tumor. Molecular pathology looking into B-cell monoclonality, and immunohistologic analysis is usually needed to confirm the characteristics of PFL. Especially, $\mathrm{Bcl} 2$ is found negative in pediatric type $\mathrm{FL}$.

Most patients require removal of the mass combined with superficial parotidectomy due to the obscure preoperative diagnosis. As Park et al. [10] stated in their case report of parotid lymphoma, the standard surgical procedure for PFL requires en bloc excision of the mass with superficial parotidectomy. This is because direct infiltration into the parotid gland parenchyme is occasionally found in postoperative pathology, although PFL is not a true parotid gland neoplasm $[4,11]$. Due to the patient's young age and well-differentiated cellular morphology, local radiotherapy rather than systemic chemotherapy was chosen for adjuvant treatment. Adjuvant radiotherapy is considered as an effective adjuvant treatment strategy in early stage local lymphomas [10,12].

Postoperative clinical course of PFL after surgical removal is excellent. Most PFLs are low-grade lymphomas without involvement of other nodal sites. Our case also exhibited no remote nodal disease on PET-CT. Systemic chemotherapy or radiation therapy is administrated according to the treatment sensitivity of the individual lymphoma. Our patient received 12 courses of radiation for prevention of local recurrence and is currently disease free.

\section{CONFLICT OF INTEREST}

No potential conflict of interest relevant to this article was reported.

\section{PATIENT CONSENT}

The parents of the patient provided written informed consent for the publication and the use of his images.

\section{ORCID}

Jangyoun Choi https://orcid.org/0000-0002-5165-8414

Hyuk Joon Choi https://orcid.org/0000-0001-9681-628X

Kwangil Yim https://orcid.org/0000-0001-8767-9033

Ho Kwon https://orcid.org/0000-0001-7471-0804

Jun Hee Byeon https://orcid.org/0000-0002-7892-6239

Sung-No Jung https://orcid.org/0000-0002-0419-4717 


\section{REFERENCES}

1. Lennon P, Silvera VM, Perez-Atayde A, Cunningham MJ, Rahbar R. Disorders and tumors of the salivary glands in children. Otolaryngol Clin North Am 2015;48:153-73.

2. Orvidas LJ, Kasperbauer JL, Lewis JE, Olsen KD, Lesnick TG. Pediatric parotid masses. Arch Otolaryngol Head Neck Surg 2000;126:177-84.

3. Andola SK, Masgal MM, Reddy RM. Diffuse large B-cell lymphoma of the parotid gland: cytological, histopathological, and immunohistochemical features: a rare case report. J Cytol 2016; 33:226-8

4. Lee HG, Lee JY, Song JM. Malignant lymphoma on parotid gland: a clinical case. J Korean Assoc Oral Maxillofac Surg 2017;43:138-43.

5. Shum JW, Emmerling M, Lubek JE, Ord RA. Parotid lymphoma: a review of clinical presentation and management. Oral Surg Oral Med Oral Pathol Oral Radiol 2014;118:e1-5.

6. Louissaint A Jr, Ackerman AM, Dias-Santagata D, Ferry JA, Hochberg EP, Huang MS, et al. Pediatric-type nodal follicular lymphoma: an indolent clonal proliferation in children and adults with high proliferation index and no BCL2 rearrangement. Blood 2012;120:2395-404.

7. Cho G, Suh IS, Tak KS, Park YK, Ko EY, Sung HM, et al. Primary parotid non-Hodgkin's lymphoma: a case report. J Korean Cleft Palate Craniofac Assoc 2010;11:99-102.

8. Yang KS, Kang SY, Shim JY, Park J, Cho SH. A case of primary malignant lymphoma of the parotid gland. J Korean Cleft Palate Craniofac Assoc 2002;3:77-81.

9. Perkins SL, Gross TG. Pediatric indolent lymphoma: would less be better? Pediatr Blood Cancer 2011;57:189-90.

10. Park ES, Park SJ, Lee YM, Yang SJ, Park CS, Kim CJ. A case of malignant lymphoma of the parotid gland. J Korean Soc Plast Reconstr Surg 1997;24:201-11.

11. Rosenblatt SD, Wolter NE, Siegele B, Brodsky JR. Primary parotid lymphoma presenting as a recurrent cystic mass: a case report. Laryngoscope 2018;128:998-1001.

12. Filippi AR, Ciammella P, Ricardi U. Limited stage follicular lymphoma: current role of radiation therapy. Mediterr J Hematol Infect Dis 2016;8:e2016041. 